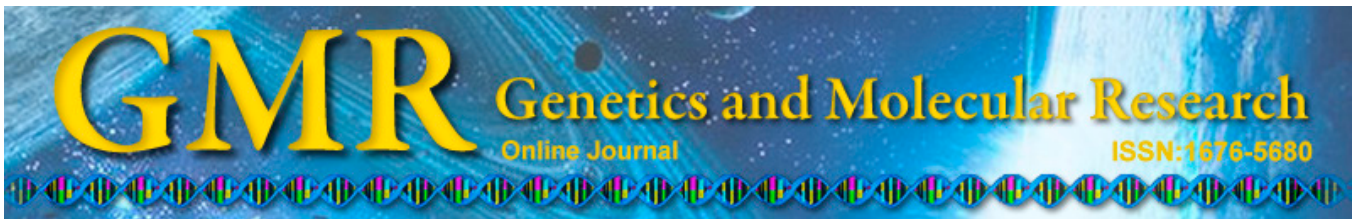

\title{
Roles of GILZ in protein metabolism of L6 muscle cells exposed to serum from septic rats
}

\author{
J. Xiong ${ }^{1,2 *}$, L. Xu ${ }^{1,2 *}$, W.M. Qu ${ }^{1,2}$, Z.L. Li ${ }^{3}$, Z.H. Shang ${ }^{1,2}$, Y.H. Li ${ }^{1}$, \\ S.H. Yang ${ }^{1,2}$ and Z.H. Yang ${ }^{1,2}$ \\ ${ }^{1}$ Three Gorges University People's Hospital, \\ The First People's Hospital of Yichang City, Yichang, China \\ ${ }^{2}$ Three Gorges University School of Medicine, Yichang, China \\ ${ }^{3}$ Changyang County People's Hospital, Yichang, China \\ *These authors contributed equally to this study. \\ Corresponding author: Z.H. Yang \\ E-mail: xiajinglinzs@126.com
}

Genet. Mol. Res. 13 (4): 8209-8219 (2014)

Received April 14, 2014

Accepted August 21, 2014

Published October 8, 2014

DOI http://dx.doi.org/10.4238/2014.October.8.2

\begin{abstract}
Sepsis is a complex inflammatory response to infection, associating with dramatic metabolic disorders. Although the mechanisms of immune response during sepsis have been largely clarified, current studies rarely pay attention to the disordered protein metabolism in sepsis. In this study, L6 rat skeletal muscle cells treated with serum from septic rats were used as an in vitro model for sepsislike condition in skeletal muscle. We found that the expression of glucocorticoid-induced leucine zipper (GILZ) positively correlates with glucocorticoid receptor and negatively correlates with myosin heavy chain expression in L6 muscle cells upon septic serum induction. Moreover, we propose that GILZ may associate with cytokines such as TNF- $\alpha$, IL- $1 \beta$ as well as IL-10 to cooperatively modulate the glucocorticoid/glucocorticoid receptor-mediated regulation of protein metabolism during sepsis. So the present study provides a new approach and theoretical basis for further studies on the regulation of
\end{abstract}


protein metabolism of skeletal muscle during sepsis.

Key words: GILZ; Glucocorticoid; Cytokines; Protein metabolism; Sepsis

\section{INTRODUCTION}

Sepsis and its concomitant multiple organ dysfunction syndrome are common complications in patients who have been subjected to severe trauma, major surgery, and infection, and are the leading causes of death in these patients (Annane et al., 2005). During sepsis, the body undergoes an extensive and complex inflammatory response, as well as a series of dramatic changes in metabolism. One of the most important metabolic changes is that proteins are presented in a high catabolic state (Lecker et al., 1999). In particular, protein catabolism in skeletal muscle is the most obvious change, clinically manifested as muscle atrophy and exhaustion. The sustained consumption of skeletal muscle protein, protein mis-folding, and the negative nitrogen balance not only seriously affect body repair but also affect the function of the immune system, and are closely associated with hypoimmunity in the anaphase of sepsis, which has a serious impact on the patient's prognosis (Hasselgren, 2000). Thus, redressing the high status of protein decomposition to reduce protein consumption will greatly help to reduce complications and mortality, thereby improving the prognosis of sepsis patients.

Activation of the ubiquitin-proteasome system is the major factor in protein degradation in skeletal muscle during sepsis. Inflammatory factors such as TNF- $\alpha$ and IL-1 $\beta$, glucocorticoid (GC), nuclear factor- $\mathrm{kB}(\mathrm{NF}-\mathrm{\kappa B})$, and oxygen-free radicals are involved in the activation of this pathway (Lecker et al., 1999; Powers et al., 2010). GC is one of the body's main anti-inflammatory molecules. It is induced under stress and has an extensive and strong anti-inflammatory effect. It also promotes protein degradation and inhibits protein synthesis (Sen et al., 1997; Seene et al., 2003; Presman et al., 2010; Smith et al., 2010). GC functions in combination with the cytoplasmic glucocorticoid receptor (GR) and the biological effects of GC directly depend on the expression levels of the GR protein (McNally et al., 2000). Combined with GC, the GR protein goes into the nucleus as a dimmer and recruits co-activators, associating with the glucocorticoid-response element to regulate several genes' transcription, thereby exerting its biological effects. A variety of cells exhibit low reactivity or no reactivity upon GC stimulation, which is referred to as GC resistance. Our previous studies found that reduced expression of GR was responsible for GC resistance (Li et al., 2006; Yang et al., 2006). GC expression increases significantly during the process of sepsis, and unlike most types of cells in which GR expression decreases upon GC activation, the expression and binding activity of GR in skeletal muscle increase reversibly, retaining a high affinity for GC and thus maintaining a high state of protein catabolism (Sun et al., 2002). In addition, GR expression also increases in L6 skeletal muscle cells in vitro when treated with serum from septic rats. However, downregulation of GR was observed in cells cultured in the resting state (Dekelbab et al., 2007). The findings above suggest that GC levels rise in the body during sepsis, but GC resistance appears in inflammatory cells, thus anti-inflammatory factor GC does not suppress the excessive inflammatory response. However, GC still selectively promotes protein catabolism and inhibits protein synthesis in skeletal muscle.

Glucocorticoid-induced leucine zipper (GILZ) was initially separated and identified as a dexamethasone (DEX)-sensitive gene from a thymocyte DNA subtractive library (Smit 
et al., 2005). GILZ is widely expressed in many organs such as the heart, brain, lung, kidney, spleen, and skeletal muscle, as well as in a variety of cells such as bone marrow cells, monocytes, mature B-lymphocytes, and T-lymphocytes (Soundararajan et al., 2005). Upon GC stimulation, expression of GILZ increases in lymphocytes, especially in monocytes and macrophages (Chen et al., 2006). GILZ may mediate the activation of T-cell receptor, inhibit the expression of Fas/FasL and IL-2 in lymphocytes, modulate T-cell activation and apoptosis, inhibit the activation of Raf-1, and be involved in the stress reaction of endothelial cells (Bhalla et al., 2006; Brennan and Fuller, 2006; Zhao et al., 2006; Reineke et al., 2007). Previous studies found that GILZ may promote the expression and activation of GR by weakening the inhibitory effects of transcription inhibitors and pro-inflammatory cytokines on GR, thereby reducing to some degree GC resistance (Sun et al., 2002). It has been determined that GILZ can inhibit skeletal muscle differentiation and mediate the roles of $\mathrm{GC}$ in inhibiting generation of skeletal muscle (Bruscoli et al., 2010).

Treatment of sepsis is still recognized as a medical challenge and most current studies focus on the implementation of anti-inflammatory mediators to modify inflammatory response in sepsis, while few studies pay attention to the pathogenesis of metabolic disorders in sepsis and the relevant interventions. In fact, metabolic disorders play an extremely important role in the development and outcomes of sepsis. In the present study, we explore the roles of GILZ in the regulation of metabolic disorders in sepsis, possibly providing a new approach and an effective therapeutic target for improving the therapeutic effect and the prognosis of sepsis patients.

\section{MATERIAL AND METHODS}

\section{Cell culture}

The rat skeletal muscle cell line L6 was purchased from the American Type Culture Collection (ATCC, USA) and plated at low density on 10-cm diameter culture dishes. Cells were grown in Dulbecco's modified Eagle's medium (Gibco) containing 10\% (v/v) charcoal stripped fetal bovine serum (HyClone, Logan, UT, USA) supplemented with $2 \mathrm{mM}$ L-glutamine (Gibco), $100 \mu \mathrm{g} / \mathrm{mL}$ sodium pyruvate (Sigma, USA), $100 \mathrm{U} / \mathrm{mL}$ penicillin, and $100 \mu \mathrm{g} /$ $\mathrm{mL}$ streptomycin (Invitrogen, USA) in a humidified atmosphere of $5 \% \mathrm{CO}_{2}$ at $37^{\circ} \mathrm{C}$.

\section{3-(4,5-dimethylthiazol-2-yl)-2,5-diphenyltetrazolium bromide (MTT) assay}

Cell proliferation in vitro was analyzed by MTT assay (Sigma) according to the manufacturer instructions. In brief, L6 cells seeded on 96-well plates in quadruplicate at a density of $1 \times 10^{4}$ cells per well were routinely cultured for $24 \mathrm{~h}$ and then replaced by $100 \mu \mathrm{L}$ fresh medium containing different concentrations of serum from septic rats, and incubated in a humidified incubator with $5 \% \mathrm{CO}_{2}$ at $37^{\circ} \mathrm{C}$. After 6,24 , and $48 \mathrm{~h}, 20 \mu \mathrm{L} 5 \mathrm{mg} / \mathrm{mL}$ MTT dissolved in PBS was added into each well and incubated for another $4 \mathrm{~h}$, followed by the addition of 150 $\mu \mathrm{L}$ dimethyl sulfoxide and incubation for $30 \mathrm{~min}$ at room temperature for color development. The absorbance values were determined using a 492-nm filter on a microplate reader (Tecan, Grödig, Austria). The experiment was performed more than three times and the inhibitory rates of L6 cells were determined as follows: (mean OD value of control - mean OD value of treatment group) / mean OD value of control x $100 \%$. 


\section{Cell induction}

Sepsis was induced in Sprague-Dawley rats and the serum was acquired from those septic rats as described previously (Sun et al., 2002). All animal work was carried out in accordance with the guidelines of the Experimental Center of the College of China Three Gorges University. L6 rat skeletal cells were cultured in fresh medium supplemented with serum from septic rats, and induced with DEX, DEX+TNF- $\alpha$, DEX+IL-1 $\beta$, or DEX+IL-10 for relative hours.

\section{RNA extraction and real-time PCR}

Total RNA was extracted from L6 myocyte cells using TRIzol reagent (Invitrogen) according to manufacturer instructions. RNA quality was tested by the absorbance ratio at $\mathrm{A}_{260 / 280}$ and $2 \mu \mathrm{L}$ total RNA was reversely transcribed into cDNA using an All-in-One ${ }^{\mathrm{TM}}$ First Strand cDNA Synthesis Kit (GeneCopoeia ${ }^{\mathrm{TM}}$, AORT-100). Then, $1 \mu \mathrm{L}$ cDNA was acquired for real-time PCR (qPCR) amplification with All-in-One ${ }^{\mathrm{TM}}$ qPCR Master Mix (GeneCopoeia ${ }^{\mathrm{TM}}$, AOPR-1200) using the following procedures: Step 1: $95^{\circ} \mathrm{C}$ for $3 \mathrm{~min}$; Step 2: $95^{\circ} \mathrm{C}$ for $15 \mathrm{~s}$; Step 3: $60^{\circ} \mathrm{C}$ for $20 \mathrm{~s}$; Step $4: 72^{\circ} \mathrm{C}$ for $20 \mathrm{~s}$, run 40 cycles. The PCR primers were designed with the Primer 5.0 software and are shown in Table 1.

Table 1. Primers for real-time PCR.

\begin{tabular}{lll}
\hline Rat gene & Forward primers $\left(5^{\prime}-3^{\prime}\right)$ & Reverse primers $\left(5^{\prime}-3^{\prime}\right)$ \\
\hline$\beta$-actin & CGTTGACATCCGTAAAGACCTC & TAGGAGCCAGGGCAGTAATCT \\
GR & TCAACGTCTGCAACTGGGTG & GGTGCTTTGGTCTGTGGGAT \\
GILZ & AGCAGCCACTCAAACCAACC & AACGGAAACCACATCCCCTC \\
\hline
\end{tabular}

\section{Western blot analysis}

L6 myocyte cells cultured with serum from septic rats for 6, 24, and $48 \mathrm{~h}$ were lysed with lysis buffer (20 mM Tris-HCl, pH 7.4, $150 \mathrm{mM} \mathrm{NaCl}, 2$ mM EGTA) containing 1\% Triton $\mathrm{X}-100,1 \mathrm{mM}$ PMSF, $10 \mathrm{nM}$ microcystin, $1 \mu \mathrm{g} / \mathrm{mL}$ aprotinin and $1 \mu \mathrm{g} / \mathrm{mL}$ leupeptin. Equal amounts of protein were separated by $10 \%$ SDS-PAGE and then transferred to nitrocellulose filter membrane (Amersham, USA). After blocking with 1\% BSA in TBST ( $0.05 \%$ Tween 20 in Tris-buffered saline) for $1 \mathrm{~h}$ at room temperature, the membranes were separately incubated overnight with anti- $\beta$-actin (Cell Signaling) at a 1:1000 dilution, anti-GR (Cell Signaling) at a 1:1000, and anti-GILZ (Santa Cruz) at a 1:100. After extensive washing with TBST, membranes were incubated with HRP-conjugated secondary antibodies (Jackson ImmunoResearch) for $1 \mathrm{~h}$ at room temperature. Then, membranes were visualized using the Pierce ECL kit (Pierce Biotechnology, Inc.) and the results were analyzed with the ImageJ software (NIH Image).

\section{Statistical analysis}

All data were analyzed using the SPSS11.5 statistics software package. Differences among the groups were determined by one-way analysis of variance (ANOVA) and correlation analyses of the expression of GILZ and GR, as well as MHC, were performed using the Spearman rank correlation coefficient. $\mathrm{P} \leq 0.05$ was considered to be statistically significant. 


\section{RESULTS}

\section{Septic rat serum prevents L6 muscle cell growth}

To survey the protein metabolism state in skeletal muscle during sepsis, we used L6 rat myocyte cells induced with serum from septic rats as an in vitro model for sepsis-like conditions in skeletal muscle. When L6 skeletal muscle cells were exposed to septic rat serum, the cell growth was notably inhibited, as observed by light microscopy. Along with longer incubation periods in culture medium containing septic rat serum, the inhibitory effects on cell growth became more and more obvious, as far fewer adherent cells were observed (Figure 1A). To quantify the cell growth state, we performed MTT assays to determine the inhibitory rates of L6 cells upon induction with different concentrations of septic rat serum for 6, 24, and $48 \mathrm{~h}$. As shown in Figure 1B, higher concentrations of septic serum exhibited much more significant inhibitory effects on cell growth. Moreover, along with longer induction periods with septic serum, the inhibitory rates increased more (Figure 1B). For incubation with $5 \mu \mathrm{L}$ septic rat serum in $2.5 \mathrm{~mL}$ culture medium for 6,24 , and $48 \mathrm{~h}$, the inhibitory rates were about 29, 43, and 53\%, respectively (Figure 1B).

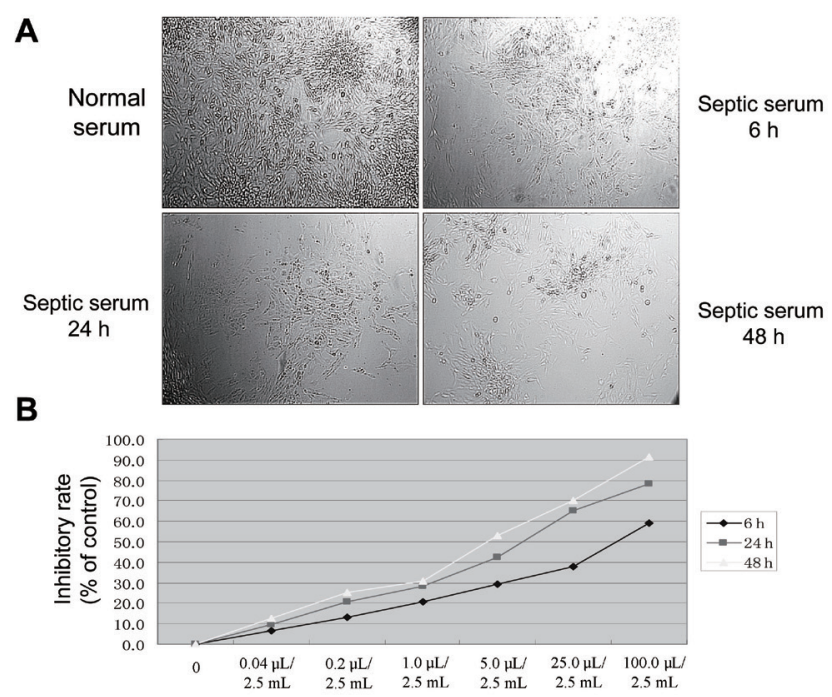

Figure 1. Septic rat serum inhibits L6 cell proliferation in vitro. A. Light microscopy observation of L6 cells cultured in medium containing serum from normal rats or septic rats for 6,24 , and $48 \mathrm{~h}$. B. Statistical analysis of the in vitro proliferation of L6 cells treated with different concentration of serum from septic rats for 6, 24, and 48 h. $* \mathrm{P}<0.05, * * \mathrm{P}<0.01, \triangle \mathrm{P}<0.05, \triangle \triangle \mathrm{P}<0.01$ compared with the control group.

\section{GILZ positively correlates with upregulated GR expression in L6 muscle cells upon septic rat serum induction}

It has been documented that GILZ is involved in the roles of GC in regulating generation and differentiation of skeletal muscle (Bruscoli et al., 2010). To clarify the roles of GILZ in GC-mediated protein consumption in skeletal muscle during sepsis, we inspected the mRNA expression levels of GILZ and GR by qRT-PCR in L6 rat muscle cells upon induction with serum from septic rats and treatment with DEX, as well as several cytokines. The relative mRNA expression levels of GILZ and GR compared with the control group are presented in 
Table 2, and the results of the statistical analysis are shown in Figure 2A and B. Six hours after induction with septic serum, GILZ and GR mRNA expression in L6 cells of different groups slightly increased. However, 24 and $48 \mathrm{~h}$ later, both GILZ and GR mRNA expression levels increased significantly in those groups compared with the control group $(\mathrm{P}<0.01$; Figure $2 \mathrm{~A})$, and the expression of GILZ positively correlated with GR $(\mathrm{r}=0.904, \mathrm{P}<0.01)$. Upon induction with septic serum for $24 \mathrm{~h}$, GR mRNA expression in the PBS treatment group was moderately upregulated compared with the control group $(\mathrm{P}<0.05)$, but GR expression increased notably in DEX, DEX+TNF- $\alpha$, DEX+IL-1 $\beta$, and DEX+IL-10 groups $(\mathrm{P}<0.01)$, and GILZ expression positively correlated with GR $(r=0.909, \mathrm{P}<0.01$; Figure $2 \mathrm{~B})$. However, the GR mRNA expression levels in L6 cells cultured with serum from normal rats were obviously downregulated in DEX, DEX+TNF- $\alpha$, and DEX+IL-1 $\beta$ groups compared with the control group $(\mathrm{P}<0.01)$ and GILZ expression positively correlated with GR $(r=0.671, \mathrm{P}<0.01)$, while no notable changes of GR expression were observed in the PBS and DEX+IL-10 groups (Figure 2B).

Table 2. Real-time RT-PCR analysis of the mRNA expression levels of GILZ and GR in different treatment groups.

\begin{tabular}{|c|c|c|c|c|c|c|c|c|c|c|}
\hline \multirow[t]{2}{*}{ Groups } & \multicolumn{2}{|c|}{ DEX } & \multicolumn{2}{|c|}{$\mathrm{DEX}+\mathrm{TNF}-\alpha$} & \multicolumn{2}{|c|}{ DEX+IL-1 $\beta$} & \multicolumn{2}{|c|}{ DEX+IL-10 } & \multicolumn{2}{|c|}{ PBS } \\
\hline & GR & GILZ & GR & GILZ & GR & GILZ & GR & GILZ & GR & GILZ \\
\hline \multicolumn{11}{|c|}{ Septic serum } \\
\hline $6 \mathrm{~h}$ & 2.42 & 1.55 & 1.74 & 1.31 & 1.35 & 1.21 & 2.52 & 3.60 & & \\
\hline $24 \mathrm{~h}$ & 6.67 & 3.83 & 4.43 & 2.44 & 3.29 & 2.04 & 8.85 & 9.68 & 2.22 & 1.59 \\
\hline $48 \mathrm{~h}$ & 7.31 & 5.28 & 5.06 & 3.50 & 3.90 & 2.56 & 8.99 & 10.54 & & \\
\hline \multicolumn{11}{|c|}{ Normal serum } \\
\hline $24 \mathrm{~h}$ & 0.45 & 0.96 & 0.23 & 0.93 & 0.12 & 0.75 & 1.03 & 1.64 & 0.98 & 1.04 \\
\hline Control & & & & & & & & & 1.00 & 1.00 \\
\hline
\end{tabular}

Septic serum group: L6 cells were treated with serum from septic rats. Normal serum group: L6 cells were treated with serum from normal rats. Control group: L6 cells were cultured routinely, as described in Material and Methods. L6 cells in the different groups were treated with PBS or DEX, DEX+TNF- $\alpha$, DEX+IL- $1 \beta$, and DEX+IL-10 for different times. Then, mRNA expressions of GR and GILZ were analyzed by real-time RT-PCR.

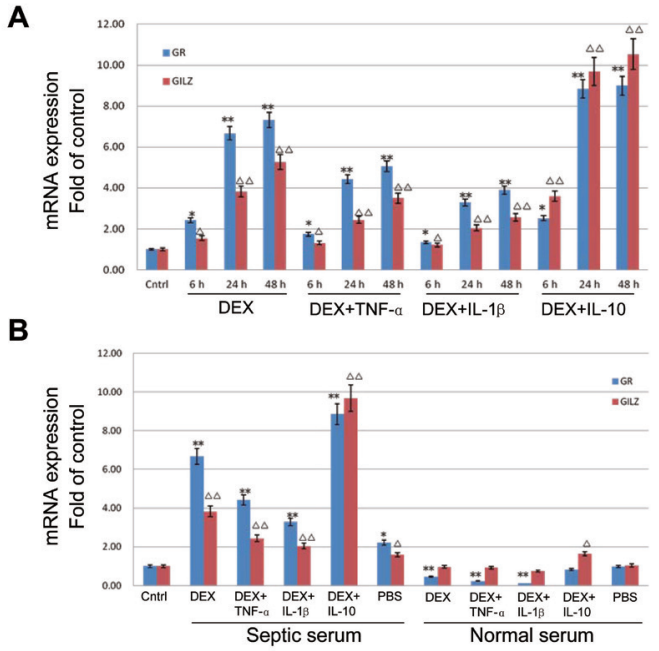

Figure 2. mRNA expression levels of GILZ positively correlate with upregulated GR mRNA expression upon septic serum induction. A. Statistical analysis of the mRNA expression levels of GILZ and GR in L6 cells induced with serum from septic rats and treated with DEX, DEX+TNF- $\alpha$, DEX+IL-1 $\beta$, and DEX+IL-10 for 6, 24, and $48 \mathrm{~h}$. B. Statistical analysis of the mRNA expression levels of GILZ and GR in L6 cells induced with serum from normal rats or septic rats and treated with PBS, DEX, DEX+TNF- $\alpha, \mathrm{DEX}+\mathrm{IL}-1 \beta$, and DEX+IL-10 for $24 \mathrm{~h} . * \mathrm{P}<0.05$, **P $<0.01, \triangle \mathrm{P}<0.05, \triangle \triangle \mathrm{P}<0.01$ compared with the control group. 


\section{GILZ negatively correlates with downregulated MHC expression in L6 muscle cells upon septic rat serum induction}

MHC expression is recognized as a marker to indicate the protein metabolism state in skeletal muscle during sepsis (Seene et al., 2003). To clarify the roles of GILZ in GC-mediated protein consumption in skeletal muscle during sepsis, we inspected protein expression of GILZ, GR, and MHC by Western blot in L6 rat muscle cells upon induction with serum from septic rats and treatment with DEX, as well as several cytokines. The Western blot results are presented in Figure 3A and the results of the statistical analyses are shown in Figure 3B. Consistent with previous qRT-PCR results, upon induction with septic serum for $24 \mathrm{~h}$, the GILZ protein expression levels in DEX, DEX+TNF- $\alpha$, DEX+IL- $1 \beta$, and DEX+IL-10 groups were significantly upregulated compared with the control group $(\mathrm{P}<0.05)$. GR expression increased much more notable $(\mathrm{P}<0.01)$ and GILZ protein expression positively correlated with $\mathrm{GR}(\mathrm{r}=0.947, \mathrm{P}<0.01$; Figure 3B). However, MHC protein expression notably decreased in those treatment groups as well as in the PBS group $(\mathrm{P}<0.01)$ and GILZ protein expression negatively correlated with MHC ( $\mathrm{r}$ $=-0.747, \mathrm{P}<0.01 ;$ Figure $3 \mathrm{~B}$ ). The GR protein expression levels in L6 cells cultured with serum from normal rats were obviously downregulated in DEX, DEX+TNF- $\alpha$, and DEX+IL- $1 \beta$ groups compared with the control group $(\mathrm{P}<0.01)$ and GILZ protein expression positively correlated with GR $(\mathrm{r}=0.955, \mathrm{P}<0.01)$, while no notable changes in GR protein expression were observed in the PBS and DEX+IL-10 groups (Figure 3B). In addition, there were no obvious changes of $\mathrm{MHC}$ protein expression in those groups compared to the control group (Figure 3B).
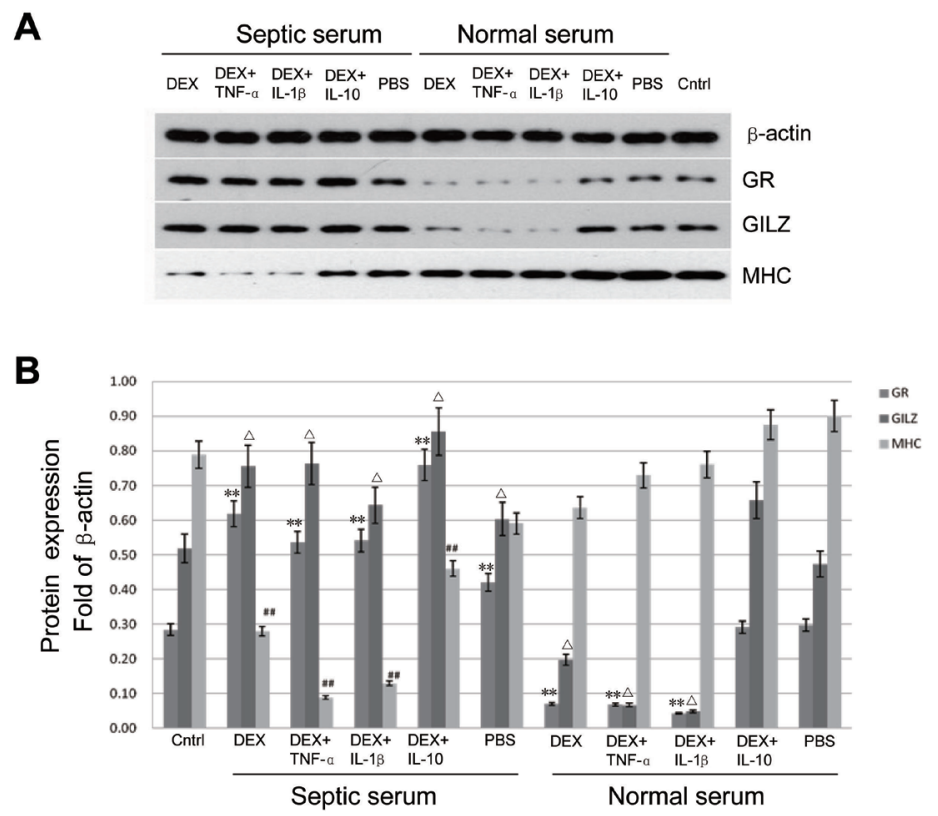

Figure 3. Protein expression levels of GILZ negatively correlate with downregulated MHC protein expression upon septic serum induction. A. Western blot analysis of the expression of GILZ, GR and MHC in L6 cells induced with serum from normal rats or septic rats and treated with PBS, DEX, DEX+TNF- $\alpha$, DEX+IL- $1 \beta$, and DEX+IL-10 for $24 \mathrm{~h}$. B. Statistical analysis of the protein expression levels as described in A. ${ }^{* * P}<0.01,{ }^{\Delta \mathrm{P}}<0.05,{ }^{\#} \mathrm{P}<0.01$ compared with the control group. 


\section{GILZ coordinates with cytokines to upregulate GR and downregulate MHC expression in L6 muscle cells exposed to septic rat serum}

Sepsis is a complex inflammatory response to infection, and many inflammatory cytokines such as TNF- $\alpha$ and IL-1 $\beta$ are involved in GC-induced effects in sepsis (Lecker et al., 1999; Hasselgren, 2000). To analyze the relationships between GILZ and those cytokines in regulating GR and MHC expression in L6 muscle cells exposed to septic serum, Western blot assays were performed to examine protein expression of GILZ, GR and MHC in L6 cells cultured in medium containing septic rat serum and DEX together with relative cytokines for 6,24 , and $48 \mathrm{~h}$. Upon induction with septic serum for 24 and $48 \mathrm{~h}$, the GILZ protein expression levels in DEX and DEX+TNF- $\alpha$ groups were significantly upregulated compared with the control group $(\mathrm{P}<0.01)$ and $\mathrm{GR}$ expression increased much more notably $(\mathrm{P}<0.01)$. GILZ protein expression positively correlated with GR $(r=0.753, \mathrm{P}<0.01$; Figure 4A and $\mathrm{B})$. However, MHC protein expression notably decreased in those treatment groups $(\mathrm{P}<0.01)$ and GILZ protein expression negatively correlated with MHC $(r=-0.791, P<0.01$; Figure 4B). Upon induction with septic serum for 24 and $48 \mathrm{~h}$, both the GILZ and GR protein expression levels in DEX+IL-1 $\beta$ and DEX+IL-10 groups increased significantly compared with the control group $(\mathrm{P}<0.05)$, and GILZ protein expression positively correlated with GR $(\mathrm{r}=0.972$, $\mathrm{P}<0.01$; Figure $4 \mathrm{C}$ and $\mathrm{D}$ ). However, MHC protein expression notably decreased in those treatment groups $(\mathrm{P}<0.05$; Figure $4 \mathrm{D})$.

A

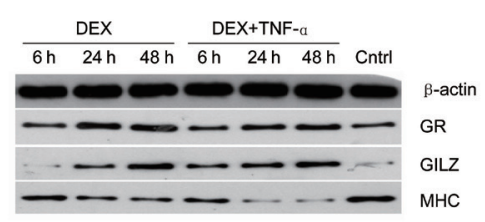

B

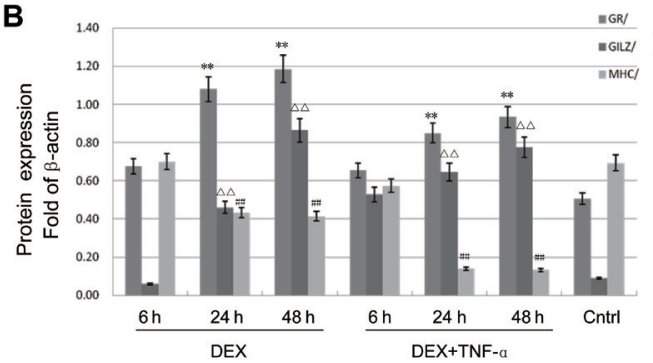

C
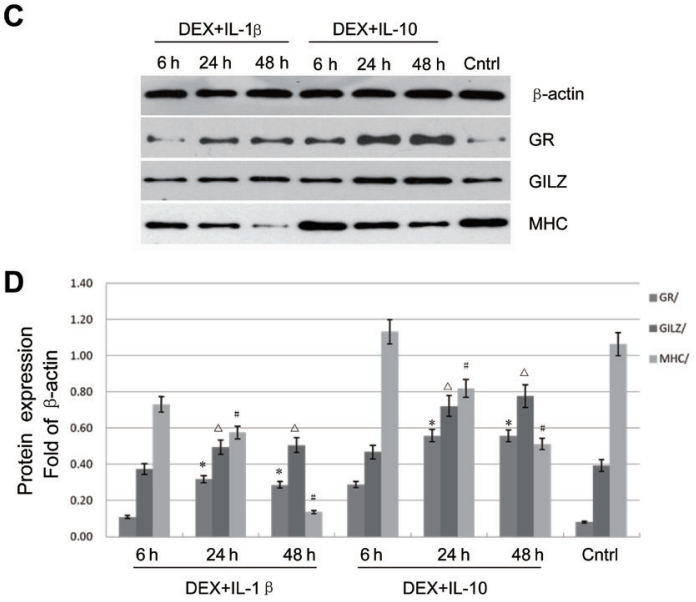

Figure 4. A. Western blot analysis of the expression of GILZ, GR and MHC in L6 cells induced with serum from septic rats and treated with PBS, DEX, and DEX+TNF- $\alpha$ for 6,24 , and $48 \mathrm{~h}$. B. Statistical analysis of the protein expression levels as described in A. C. Western blot analysis of the expression of GILZ, GR, and MHC in L6 cells induced with serum from septic rats and treated with PBS, DEX+IL-1 $\beta$, and DEX+IL-10 for 6, 24, and $48 \mathrm{~h}$. D. Statistical analysis of the protein expression levels as described in $\mathbf{C} .{ }^{*} \mathrm{P}<0.05,{ }^{*} * \mathrm{P}<0.01,{ }^{\Delta} \mathrm{P}<0.05,{ }^{\Delta \Delta} \mathrm{P}<0.01$, ${ }^{\#} \mathrm{P}<0.05,{ }^{\#} \mathrm{P}<0.01$ compared with the control group.

\section{DISCUSSION}

It has been reported that GR expression is downregulated in L6 myocyte cells when treated with the synthetic glucocorticoid, DEX (Dekelbab et al., 2007). Consistent with this, 
our results showed that when L6 cells were cultured in a medium containing serum from normal rats together with DEX, DEX+TNF- $\alpha$, and DEX+IL- $1 \beta$ for $24 \mathrm{~h}$, both mRNA and protein expression of GR decreased significantly compared with the control group $(\mathrm{P}<0.05$; Figures $2 \mathrm{~B}$ and $3 \mathrm{~B}$ ). However, both mRNA and protein expression levels of GR were largely upregulated in cells of those groups exposed to serum from septic rats $(\mathrm{P}<0.05$; Figures $2 \mathrm{~B}$ and $3 \mathrm{~B})$. The results above suggest that the muscle cells are usually GC-resistant in the resting state, but sensitive to GC induction during sepsis.

GILZ is one of the GC-induced genes and appears to be a critical factor in the anti-inflammatory effects of GCs (Ayroldi and Riccardi, 2009). It has been reported that the synthetic glucocorticoid, DEX, may promote the expression and activation of GR in septic muscle (Sun et al., 2002), so the DEX-induced gene GILZ may be involved in GC-mediated regulation of sepsis in skeletal muscle. In the present study, we found that mRNA and protein expression of GILZ positively correlated with GR $(0<\mathrm{r}<1, \mathrm{P}<0.01)$ (Figures $2 \mathrm{~A}, \mathrm{~B}, 3 \mathrm{~B}, 4 \mathrm{~A}$, and D). The GILZ-mediated regulation of GR may be partially achieved by modulating the activation of $\mathrm{NF}-\kappa \mathrm{B}$, an antagonist of GR, as previous studies have found that GILZ could inhibit the activity of NF- $\kappa$ B (Ayroldi and Riccardi, 2009). However, further studies are required to clarify the mechanisms of GILZ in regulating GR expression. MHC expression is an indicator for the protein consumption in skeletal muscle during sepsis (Seene et al., 2003). As our studies have shown, there are no obvious changes of MHC expression in L6 cells cultured with serum from normal rats. However, MHC protein expression was downregulated significantly in L6 cells exposed to septic rat serum $(\mathrm{P}<0.01$; Figure $3 \mathrm{~B})$. Moreover, GILZ expression negatively correlated with MHC (Figures 3B and 4B), indicating that GILZ is involved in GC-induced protein consumption in skeletal muscle cells exposed to septic serum.

Sepsis is an inflammatory response to infection via activation of the innate immune system, involving a complex network of inflammatory mediators including cytokines such as TNF- $\alpha$ and IL-1 $\beta$, which are upstream activators of NF- $\kappa B$ (Brown and Jones, 2004). NF- $\kappa B$ is believed to be a GR antagonist and plays a major role in the acquired GC resistance in some septic patients. In our study, when L6 cells were treated with DEX+TNF- $\alpha$ or DEX+IL-1 , both mRNA and protein expression of GR generally decreased compared with cells treated with DEX and DEX+IL-10 upon septic serum induction (Table 2 and Figures 2B and 3B), suggesting that the downregulation of GR may be caused by antagonizing GR through the NF- $\kappa$ B pathway, possibly mediated by TNF- $\alpha$ and IL- $1 \beta$. The anti-inflammatory mediator IL10 also has been reported to closely associate with the process of sepsis and stimulate GILZ synthesis in some types of cell (Godot et al., 2006). In our study, when L6 cells were treated with DEX+IL-10, both mRNA and protein expression of GILZ largely increased compared with cells treated with DEX, DEX+TNF- $\alpha$, and DEX+IL-1 $\beta$ (Figures 2, 3, and 4). Meanwhile, both mRNA and protein expression of GR in cells treated with DEX+IL-10 were higher than those cells treated with DEX, DEX+TNF- $\alpha$, and DEX+IL-1 $\beta$ upon septic serum induction (Figures 2, 3, and 4). So these results indicate that IL-10-mediated upregulation of GILZ may be partially responsible for the elevated expression of GR. The pro-inflammatory cytokines TNF- $\alpha$ and IL- $1 \beta$ exert antagonistic effects against GC-mediated anti-inflammatory response, and our study found that TNF- $\alpha$ and IL- $1 \beta$ downregulated GR expression in L6 cells treated with DEX (Table 2 and Figures 2B and 3B). However, although lower GR expression was observed in TNF- $\alpha$ - and IL- $1 \beta$-treated cells compared with cells treated with DEX alone upon septic serum induction, TNF- $\alpha$ and IL-1 $\beta$ strongly promoted MHC proteolysis (Figures 2, 
3 , and 4). These results suggest that TNF- $\alpha$ and IL-1 $\beta$ may regulate muscle proteolysis by glucocorticoid-independent pathways, which is consistent with previous studies.

Taking these findings together, we hypothesize that GILZ may be sensitive to the elevated expression of GC in septic muscle, promoting the expression and activation of GR involved in GC/GR-mediated proteolysis in septic skeletal muscle. However, further experiments are required to clarify the detailed mechanisms of GILZ in regulating the protein metabolism of skeletal muscle during sepsis. Moreover, much more attention should be paid to the studies of metabolic disorders in sepsis, and this study may provide a new approach and an effective target for improving the therapeutic effect and the prognosis of sepsis patients.

\section{ACKNOWLEDGMENTS}

Research supported by the Young Scientist Project of the Health Department of Hubei Province, China (\#QJX2012-43).

\section{REFERENCES}

Annane D, Bellissant E and Cavaillon JM (2005). Septic shock. Lancet 365: 63-78.

Ayroldi E and Riccardi C (2009). Glucocorticoid-induced leucine zipper (GILZ): a new important mediator of glucocorticoid action. FASEB. J. 23: 3649-3658.

Bhalla V, Soundararajan R, Pao AC, Li H, et al. (2006). Disinhibitory pathways for control of sodium transport: regulation of ENaC by SGK1 and GILZ. Am. J. Physiol. Ren. Physiol. 291: F714-F721.

Brennan FE and Fuller PJ (2006). Mammalian K-ras2 is a corticosteroid-induced gene in vivo. Endocrinology 147: 28092816.

Brown MA and Jones WK (2004). NF-kappaB action in sepsis: the innate immune system and the heart. Front Biosci. 9: 1201-1217.

Bruscoli S, Donato V, Velardi E, Di Sante M, et al. (2010). Glucocorticoid-induced leucine zipper (GILZ) and long GILZ inhibit myogenic differentiation and mediate anti-myogenic effects of glucocorticoids. J. Biol. Chem. 285: 10385-10396.

Chen W, Rogatsky I and Garabedian MJ (2006). MED14 and MED1 differentially regulate target-specific gene activation by the glucocorticoid receptor. Mol. Endocrinol. 20: 560-572.

Dekelbab BH, Witchel SF and DeFranco DB (2007). TNF-alpha and glucocorticoid receptor interaction in L6 muscle cells: a cooperative downregulation of myosin heavy chain. Steroids 72: 705-712.

Godot V, Garcia G, Capel F, Arock M, et al. (2006). Dexamethasone and IL-10 stimulate glucocorticoid-induced leucine zipper synthesis by human mast cells. Allergy 61: 886-890.

Hasselgren PO (2000). Catabolic response to stress and injury: implications for regulation. World J. Surg. 24: 1452-1459.

Lecker SH, Solomon V, Mitch WE and Goldberg AL (1999). Muscle protein breakdown and the critical role of the ubiquitin-proteasome pathway in normal and disease states. J. Nutr. 129: 227S-237S.

Li B, Bai X and Wanh H (2006). Effect of dexamethasone on expression of glucocorticoid receptor in human monocyte cell line THP-1. J. Huazhong. Univ. Sci. Technol. Med. Sci. 26: 25-27.

McNally JG, Muller WG, Walker D, Wolford R, et al. (2000). The glucocorticoid receptor: rapid exchange with regulatory sites in living cells. Science 287: 1262-1265.

Powers SK, Duarte J, Kavazis AN and Talbert EE (2010). Reactive oxygen species are signalling molecules for skeletal muscle adaptation. Exp. Physiol. 95: 1-9.

Presman DM, Alvarez LD, Levi V, Eduardo S, et al. (2010). Insights on glucocorticoid receptor activity modulation through the binding of rigid steroids. PLoS One 5: e13279.

Reineke EL, Liu H, Lam M, Liu Y, et al. (2007). Aberrant association of promyelocytic leukemia protein-retinoic acid receptor-alpha with coactivators contributes to its ability to regulate gene expression. J. Biol. Chem. 282: 1858418596.

Seene T, Kaasik P, Pehme A, Alev K, et al. (2003). The effect of glucocorticoids on the myosin heavy chain isoforms' turnover in skeletal muscle. J. Steroid Biochem. Mol. Biol. 86: 201-206.

Sen CK, Khanna S, Reznick AZ, Roy S, et al. (1997). Glutathione regulation of tumor necrosis factor-alpha-induced NFkappa B activation in skeletal muscle-derived L6 cells. Biochem. Biophys. Res. Commun. 237: 645-649. 
Smit P, Russcher H, de Jong FH, Brinkmann AO, et al. (2005). Differential regulation of synthetic glucocorticoids on gene expression levels of glucocorticoid-induced leucine zipper and interleukin-2. J. Clin. Endocrinol. Metab. 90: 2994-3000.

Smith IJ, Alamdari N, O'Neal P, Gonnella P, et al. (2010). Sepsis increases the expression and activity of the transcription factor Forkhead Box O 1 (FOXO1) in skeletal muscle by a glucocorticoid-dependent mechanism. Int. J. Biochem. Cell. Biol. 42: 701-711.

Soundararajan R, Zhang TT, Wang J, Vandewalle A, et al. (2005). A novel role for glucocorticoid-induced leucine zipper protein in epithelial sodium channel-mediated sodium transport. J. Biol. Chem. 280: 39970-39981.

Sun X, Fischer DR, Pritts TA, Wray CJ, et al. (2002). Expression and binding activity of the glucocorticoid receptor are upregulated in septic muscle. Am. J. Physiol. Regul. Integr. Comp. Physiol. 282: R509-R518.

Yang Z, Bai X, Wang H, Li Z, et al. (2006). Correlation between endotoxin tolerance in human monocyte leukemia cell line THP-1 with glucocorticoid receptor-alpha. J. Huazhong Univ. Sci. Technol. Med. Sci. 26: 28-30.

Zhao B, Koon D and Bethin KE (2006). Identification of transcription factors at the site of implantation in the later stages of murine pregnancy. Reproduction 131: 561-571. 